\title{
Sensitivity Analysis of a Hemodynamic-based Model for Thrombus Formation and Growth
}

\author{
Gian Marco Melito', Alireza Jafarinia², Thomas Hochrainer ${ }^{2}$, Katrin Ellermann \\ ${ }^{1}$ Institute of Mechanics/University of Technology Graz \\ Kopernikusgasse 24/IV, Graz, Austria \\ gmelito@tugraz.at; ellermann@tugraz.at \\ ${ }^{2}$ Institute of Strength of Materials/University of Technology Graz \\ Kopernikusgasse 24/I, Graz, Austria \\ alireza.jafarinia@tugraz.at; hochrainer@tugraz.at
}

\begin{abstract}
Aortic dissection is a severe cardiovascular disease caused by the occurrence of a tear in the aortic wall. As a result, the blood penetrates the wall and makes a new blood channel called false lumen. The hemodynamic conditions in the false lumen may contribute to the formation of thrombi, which influence the patient diagnosis and outcomes. In this study, the focus is on a hemodynamic-based model of thrombus formation. Since the model construction presents sources of uncertainties in the model parameter, a variance-based sensitivity analysis is performed. Thrombus formation at a backwards-facing step is considered as a benchmark for the numerical simulations and sensitivity analysis. This geometry is capable of representing the main contributions of the model in thrombus formation. The study aims not only at getting a better insight into the model's structure but also at preparing model simplifications with the aim of future patient-specific simulations. A polynomial chaos expansion is employed as a surrogate model, from which the derivation of quantitative sensitivity indices is enhanced. In this study, nine model parameters are selected, whose actual values are not well known. The model responses taken into account are the maximum volume fraction of thrombosis, its time development, and the thrombus growth rate. The results show that the model lends itself to model reduction since some of the model parameters show little to no influence on the model's outputs. A threshold value related to the concentration of bounded platelets is identified as the key input parameter dominating the model predictions in the current geometry.
\end{abstract}

Keywords: Aortic Dissection, Thrombus Formation, Global Sensitivity Analysis, Backwards-Facing Step

\section{Introduction}

Aortic dissection (AD) is a disease that develops a second volume, called false lumen (FL), in the aorta. AD is classified as type A when it initiates in the ascending thoracic aorta, type B when the initial tear in the aortic wall is in the descending thoracic aorta (Stanford Classification System). Type B aortic dissection (TBAD) is a severe disease associated with high mortality, which may also lead to complications such as aortic aneurysm, rupture or malperfusion syndromes [1]. In the current study, we focus on TBAD and the role of thrombosis in the false lumen.

The haemodynamic conditions in the FL, including flow disturbance, recirculations, and significant variability in the wall shear stress (WSS) presumably contribute to the formation and growth of thrombi [2]. In [3], it is showed that partial thrombosis is associated with a higher mortality rate, whereas complete thrombosis of the FL improves patients' prognosis $[4,5]$. Up to now, it is unclear what circumstances favour thrombosis following aortic dissection. Thrombus formation models may play a vital role in the analysis of hemodynamics in cardiovascular environments.

In $[2,6]$ a haemodynamic-based model capable of predicting false lumen thrombosis in TBAD is developed. However, because the model is mostly phenomenological, the parameters of the model may not be determined from chemical or biological characteristics of the blood. Instead, the parameters will usually be obtained from inverse modelling, i.e. fitting to measured data. As suitable data is very sparse, it is of vital importance to narrow down the number of model parameters. A global sensitivity analysis is thus suggested to understand the influence of the parameters. Moreover, although the model performs well in predicting the location of thrombus formation, so far it is unable to reproduce the growth rate as observed in in-vivo and in-vitro studies. Therefore, in order to bridge the gap between numerical simulations and real-life studies, we need more insight into the model parameters and their role in thrombus growth. 
The current study aims at quantifying and reducing the uncertainty in the model. The research questions that are at the core of the study are: which input parameters influence the growth rate and final volume of the thrombus? How is the influence of the parameters distributed over the time of thrombus formation and growth? That is, if some input parameters have an active role in thrombus formation at a particular moment of the process.

A global sensitivity analysis is performed with a variance-based approach. The variance of the quantity of interest is decomposed in the sum of the space contributions of input parameters [7]. We decided to employ a variance-based approach, since knowledge regarding the model is limited, in the sense that it is unknown whether its behaviour with respect to the parameters is linear, additive, monotonic, or none of them.

\section{Thrombus formation and growth}

In this study, the thrombus formation model developed by [2] is used. It is a hemodynamic-based model in which the thrombus forms and grows mainly in areas where low shear stress and high residence time are measured. The model consists of the following equations, which are coupled with Navier-Stokes equations. High residence time $T_{\mathrm{R}}$ marks the areas where platelets spend more time [2],

$$
\frac{\partial T_{\mathrm{R}}}{\partial t}+\boldsymbol{u} \cdot \nabla T_{\mathrm{R}}=D_{\mathrm{T}_{\mathrm{R}}} \nabla^{2} T_{\mathrm{R}}+1
$$

where $\boldsymbol{u}$ denotes the velocity vector, and $D_{\mathrm{T}_{\mathrm{R}}}$ is the diffusion coefficient of the residence time. The transport equation for the concentration $c_{\mathrm{i}}$ of resting or activated platelets (RP or AP, resp.) is

$$
\frac{\partial c_{\mathrm{i}}}{\partial t}+\boldsymbol{u} \cdot \boldsymbol{\nabla} c_{\mathrm{i}}=D_{\mathrm{P}} \boldsymbol{\nabla}^{2} c_{\mathrm{i}}+s_{\mathrm{i}}, \quad \mathrm{i}=\mathrm{RP}, \mathrm{AP}
$$

Here, $D_{\mathrm{P}}$ denotes the diffusion coefficient of platelets, which is the same for resting and activated platelets. Furthermore, $s_{\mathrm{i}}$ denote reaction terms for the conversion of resting to activated platelets [2].

The so-called coagulant concentration $c$ accounts for the lumped effect of all underlying biochemical reactions in the coagulation cascade [2]. In low shear rate areas, there is a production of coagulant at the wall based on the conditions specified on the boundary, compare Eq. (4). This modified boundary condition for the flux of coagulant is taken from [6] for the backwards-facing step. The diffusion-reaction equation for the coagulant is

$$
\begin{gathered}
\frac{\partial c}{\partial t}=D_{\mathrm{c}_{\mathrm{eff}}} \nabla^{2} c+k_{\mathrm{c}} \phi_{\mathrm{th}} \phi_{\dot{\gamma}}, \text { where } \phi_{\dot{\gamma}}=\dot{\gamma}_{\mathrm{t}}^{2} /\left(\dot{\gamma}^{2}+\dot{\gamma}_{\mathrm{t}}^{2}\right), \text { and } \\
\left.D_{\mathrm{c}_{\mathrm{eff}}} \frac{\partial c}{\partial n}\right|_{\text {wall }}=\left\{\begin{array}{cl}
k_{\mathrm{c} \text {,wall }} & \text { if } \dot{\gamma}<1 \mathrm{~s}^{-1} \text { and } c_{\mathrm{BP}} \leq 200 \mathrm{nM}, \\
0 & \text { otherwise, }
\end{array}\right.
\end{gathered}
$$

where $k_{\mathrm{c}}$ is the coagulant kinetic constant, $\dot{\gamma}$ is the shear rate, and the subscript $\mathrm{t}$ denotes the threshold values. The effective coagulant diffusivity $D_{\mathrm{c}_{\mathrm{eff}}}$ is proportional to the coagulant diffusivity $D_{\mathrm{c}}$,

$$
D_{\mathrm{c}_{\mathrm{eff}}}=\phi_{\dot{\gamma}} D_{\mathrm{c}}
$$

Finally, the rate of production of bounded platelets concentration $c_{\mathrm{BP}}$ is given by

$$
\frac{\partial c_{\mathrm{BP}}}{\partial \mathrm{t}}=k_{\mathrm{BP}} \phi_{\mathrm{BP}} \phi_{\dot{\gamma}} c_{\mathrm{AP}} \text {, where } \phi_{\mathrm{BP}}=\left(c^{2} /\left(c^{2}+c_{\mathrm{t}}^{2}\right)\right)\left(T_{\mathrm{R}}^{2} /\left(T_{\mathrm{R}}^{2}+T_{\mathrm{R}_{\mathrm{t}}}^{2}\right)\right) \text {, }
$$


where $k_{\mathrm{BP}}$ is the bounded platelets reaction rate, and $c_{\mathrm{AP}}$ is the activated platelets concentration. The Navier-Stokes equation is modified to incorporate the thrombus growth [2]

$$
\rho\left[\frac{\partial u}{\partial t}+(\boldsymbol{u} \cdot \boldsymbol{\nabla}) \boldsymbol{u}\right]=-\boldsymbol{\nabla} p+\boldsymbol{\nabla} \cdot \boldsymbol{\tau}-k_{\mathrm{th}} \phi_{\mathrm{th}} \boldsymbol{u}
$$

where $\rho$ denotes the blood density, $p$ the pressure, $\boldsymbol{\tau}$ is the Cauchy stress tensor, $\phi_{\mathrm{th}}\left(c_{\mathrm{BP}}, c_{\mathrm{BP}}\right)=c_{\mathrm{BP}}^{2} /\left(c_{\mathrm{BP}}^{2}+c_{\mathrm{BPt}}^{2}\right)$ and it indicates local thrombosis as a function of the bounded-platelets concentration $c_{\mathrm{BP}} . k_{\mathrm{th}}$ is a coefficient with a sufficiently high value to stop the flow where the thrombus is formed [2]. In summary, the model controls the formation of thrombus based on shear stress, residence time, the concentration of coagulant, and bounded platelets.

\subsection{Numerical simulations}

OpenFOAM software is used for solving blood flow and thrombus formation equations. The blockMesh utility in OpenFOAM is used for generating a structured hexahedral mesh. Mesh and time-step sensitivity analysis resulted in 20000 elements and time-step of $0.005 \mathrm{~s}$. Blood is assumed as a Newtonian fluid. The thrombus formation simulation starts at 12 seconds from the steady-state flow solution. A Reynolds number of 490 is chosen to be consistent with in-vitro results in [8] and numerical simulations in [9]. In Fig. 1, the velocity magnitude contour of the steady-state solution is shown. The model predicts thrombus formation in the recirculation area behind the step, see Fig. 3.

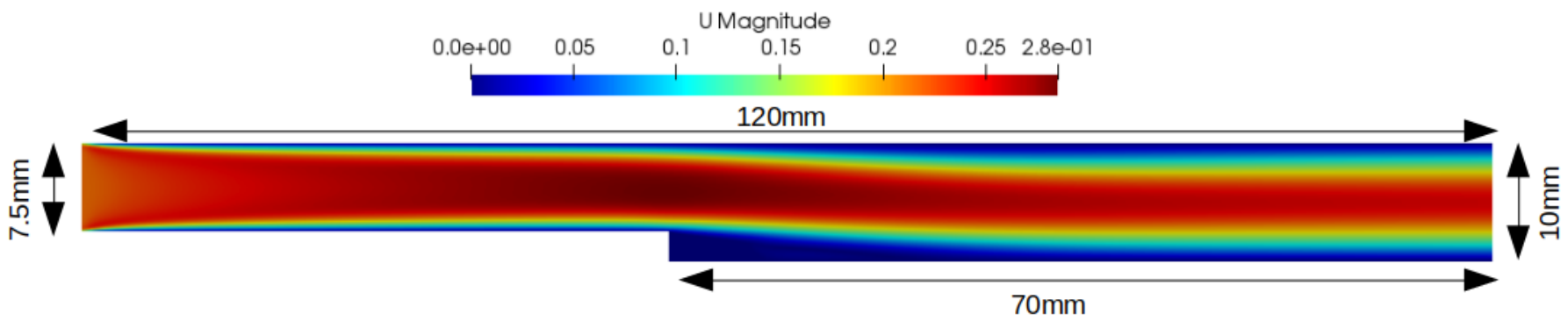

Fig. 1: Velocity magnitude contour.

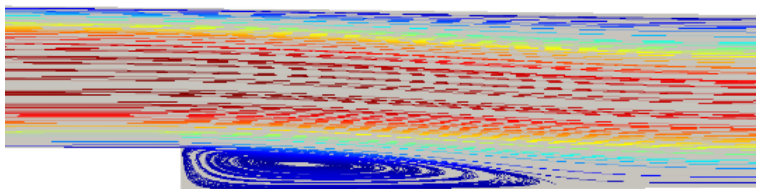

Fig. 2: Streamlines depicting recirculation at the back of the step.

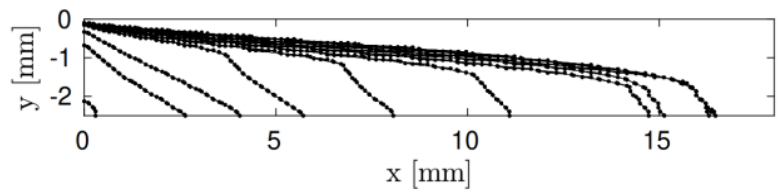

Fig. 3 Evolution of thrombus in time at the back of the step, the thrombus has reached to $16 \mathrm{~mm}$ length in $50 \mathrm{~s}$.

\section{Sensitivity analysis}

One of the most widely used technique in global sensitivity analysis is the variance-based method. Consider a quantity of interest $Y$ of a model as a function of an input random vector $\boldsymbol{x}$ of dimension $n$, i.e. $Y=f(\boldsymbol{x})=f\left(x_{1}, x_{2}, \ldots, x_{n}\right)$. Global sensitivity analysis aims to quantify the connection between the variance of the model output, given the variability of its input $x_{i}$ to make the model personalization easier.

A random variable $x_{\mathrm{i}}$ is considered to be influential (non-influential) to the model $f$ with output $Y$ if the conditional variance $\mathbb{V}\left[\mathbb{E}\left[Y \mid x_{\mathrm{i}}\right]\right]$ is larger (smaller) than the variance of the quantity of interest $\mathbb{V}[Y]$, where $\mathbb{V}[\cdot]$ and $\mathbb{E}[\cdot]$ represent the variance and the mean operators. The first-order sensitivity index (or first-Sobol index) is defined as [10] 


$$
S_{\mathrm{i}}=\frac{\mathbb{V}\left[\mathbb{E}\left[Y \mid x_{\mathrm{i}}\right]\right]}{\mathbb{V}[Y]} .
$$

The Sobol index represents the contribution of the random variable $x_{\mathrm{i}}$, for $1 \leq i \leq n$, to the change of the model output $Y$.

The total-order sensitivity index (or total-Sobol index) is defined as

$$
S_{\mathrm{i}}^{\mathrm{T}}=1-\frac{\mathbb{V}\left[\mathbb{E}\left[Y \mid \boldsymbol{x}_{\sim \mathrm{i}}\right]\right]}{\mathbb{V}[Y]}=\frac{\mathbb{E}\left[\mathbb{V}\left[Y \mid \boldsymbol{x}_{\sim \mathrm{i}}\right]\right]}{\mathbb{V}[Y]} .
$$

This index evaluates the total effect of an input parameter, by accounting for the conditional variance of the output, conditioning to all factors except the given one, $\boldsymbol{x}_{\sim \mathrm{i}}$.

The first-order index identifies the level of influence of the single parameter on the output in the analysis, but it does not give information regarding the interaction of the parameter with other variables of the input space. It allows factor prioritization setting, where a ranking of the parameters is produced. The rank indicates the level of influence on the model variation. The total-order sensitivity index specifies the influence of the input parameter on the model output and the level of interaction with other input parameters. The total-order index is used to produce the factor fixing setting. Here, the lower values are considered to decide which variable has no- or low-effect on the output. Such variables can be successively considered as model's constant.

\subsection{Polynomial Chaos Expansion}

The Sobol indices are computed from a polynomial chaos expansion (PCE) of the model [11]. The expansion will also serve as metamodel of the thrombus formation model. PCE consists of the sum of orthogonal, multivariate polynomials $\boldsymbol{\Psi}_{\alpha}$ of increasing order up to some maximal polynomial order $p$ [12]. The polynomials are multiplied by expansion coefficients $y_{\alpha}$, which can be estimated with different methods. The expansion is written as

$$
Y(\boldsymbol{x}) \approx f_{\mathrm{PCE}}(\boldsymbol{x})=\sum_{\alpha \in A} y_{\alpha} \boldsymbol{\Psi}_{\alpha}(\boldsymbol{x}),
$$

where $A$ is a set of multi-indices $\alpha$ which refer to the degree of each polynomial and each input parameter, the multivariate polynomials $\boldsymbol{\Psi}_{\alpha}$ are defined as the product of univariate polynomials of order $\alpha_{\mathrm{i}}$, i.e. $\psi_{\alpha_{\mathrm{i}}}$. The univariate polynomials are generated following the Askey scheme [13] for the composition of polynomials.

Finally, from the PCE, it is possible to estimate the two sensitivity indices as the ratio between the PCE coefficients [13]. Since the case study is dynamic, the variance of the output evolves in time. A pointwise-in-time evaluation of sensitivity indices turns out to be inaccurate in describing such evolution. Therefore, an analysis that is aware of the history of the output variability is needed. The implementation of time-dependent indices, known as generalized Sobol indices [14] read as

$$
\begin{gathered}
S_{\mathrm{i}}(t)=\frac{\int_{0}^{\mathrm{t}} \mathbb{V}_{i}\left[Y\left(x_{i}, \tau\right)\right] d \tau}{\int_{0}^{\mathrm{t}} \mathbb{V}[Y(\boldsymbol{x}, \tau)] d \tau} \approx \frac{\sum_{\alpha \in \mathrm{A}_{\mathrm{i}}} y_{\alpha}^{2}(t)}{\sum_{\alpha \in \mathrm{A}_{\mathrm{i}} ; \alpha \neq 1} y_{\alpha}^{2}(t)}=\frac{\int_{0}^{\mathrm{t}} \sum_{\alpha \in \mathrm{A}_{\mathrm{i}}} y_{\alpha}^{2}(\tau) d \tau}{\int_{0}^{\mathrm{t}} \sum_{\alpha \in \mathrm{A}_{\mathrm{i}} ; \alpha \neq 1} y_{\alpha}^{2}(\tau) d \tau}, \\
S_{\mathrm{i}}^{\mathrm{T}}(t) \approx \frac{\sum_{\alpha \in \mathrm{A}_{\mathrm{i}}^{\mathrm{T}}} y_{\alpha}^{2}(t)}{\sum_{\alpha \in \mathrm{A}_{\mathrm{i}} ; \alpha \neq 1} y_{\alpha}^{2}(t)}=\frac{\int_{0}^{t} \sum_{\alpha \in \mathrm{A}_{\mathrm{i}}^{\mathrm{T}}} y_{\alpha}^{2}(\tau) d \tau}{\int_{0}^{t} \sum_{\alpha \in \mathrm{A}_{\mathrm{i}} ; \alpha \neq 1} y_{\alpha}^{2}(\tau) d \tau} .
\end{gathered}
$$




\subsection{Application to the thrombus formation model}

The input parameters that are considered to represent uncertainty are listed in Tab. 1. To adequately cover and understand the sensitivity of chosen parameters on thrombus formation, the volume fraction of thrombosis and the thrombus growth rate are considered as the quantity of interest. The volume fraction of thrombosis of the simulation volume $V$ is defined as

$$
\bar{\phi}_{\mathrm{th}}(t)=\frac{1}{V} \int_{\mathrm{V}} \phi_{\mathrm{th}}(x, y, z, t) d V
$$

and the thrombus growth rate as:

$$
\dot{\bar{\phi}}_{\mathrm{th}}(t)=\frac{d}{d t}\left(\bar{\phi}_{\mathrm{th}}(t)\right)=\frac{1}{V} \frac{d}{d t} \int_{\mathrm{V}} \phi_{\mathrm{th}}(x, y, z, t) d V
$$

Each random variable has been classified as uniformly distributed since the knowledge about their distribution is, at the moment, unclear. This approach allows evaluating different scenarios in which the thrombus formation could be highly or mildly altered. The sample is produced with latin hypercube sampling techniques with size $N_{\mathrm{s}}$ equal to 450 . This number of simulations is bounded by the high computational cost of the model and fulfils the requirements for the construction of the PCE. The latter is solved with the regression LARS method through the Matlab toolbox UQlab [15], the degree of the polynomial is set to 3 because the convergence of sensitivity indices is achieved.

Table 1: Input parameters of the thrombus model and their probabilistic distribution used for the sensitivity analysis. All the input parameters follow a uniform probability distribution.

\begin{tabular}{lll}
\hline Parameters & Notation & PDF parameters \\
\hline Activated platelets concentration & $c_{\mathrm{AP}}$ & {$[1.00 \mathrm{e}-10,1.00 \mathrm{e}-06]$} \\
Coagulant diffusivity & $D_{\mathrm{c}}$ & {$[2.00 \mathrm{e}+04,2.00 \mathrm{e}+06]$} \\
Coagulant kinetic constant & $k_{\mathrm{c}}$ & {$[8.00 \mathrm{e}-11,8.00 \mathrm{e}-09]$} \\
Bounded platelets reaction rate & $k_{\mathrm{BP}}$ & {$[1.00 \mathrm{e}+03,1.00 \mathrm{e}+05]$} \\
Coagulant concentration threshold & $c_{\mathrm{t}}$ & {$[2.00 \mathrm{e}+03,2.00 \mathrm{e}+05]$} \\
Bounded platelets concentration threshold & $c_{\mathrm{BPt}}$ & {$[1.00 \mathrm{e}-01,3.00 \mathrm{e}+00]$} \\
Coagulant kinetic constant at the wall & $k_{\mathrm{c}, \mathrm{wall}}$ & {$[1.00 \mathrm{e}+02,1.00 \mathrm{e}+05]$} \\
Residence time threshold & $T_{\mathrm{R}}$ & {$[0.750 \mathrm{e}+13,2.25 \mathrm{e}+13]$} \\
Bounded platelets concentration threshold at the wall & $c_{\mathrm{BPbt}}$ & {$[1.00 \mathrm{e}+02,2.50 \mathrm{e}+05]$} \\
\hline
\end{tabular}

\section{Results}

Fig. 4 shows the frequency of the recorded maximum volume fraction of thrombosis. Its distribution resembles a Gamma distribution whose mean value shows that on average $3 \%$ of the simulation volume is predicted to be a thrombus. The range of the response varies from the absence of thrombus to thrombus coverage of over $20 \%$ of the volume domain.

The sensitivity indices $S_{\mathrm{i}}$ and $S_{\mathrm{i}}^{\mathrm{T}}$ for the thrombus formation model are listed in Tab. 2. By looking at the first column, one observes that the first-order sensitivity indices do not sum up to one, which occurs in the presence of non-additive model behaviour. Thus only $90 \%$ of the output variance can be attributed to a single random variable. 


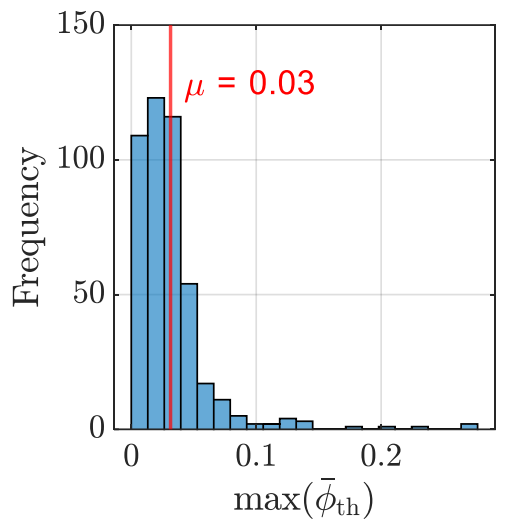

Fig. 4: Frequency histogram of the maximum volume fraction of thrombosis.

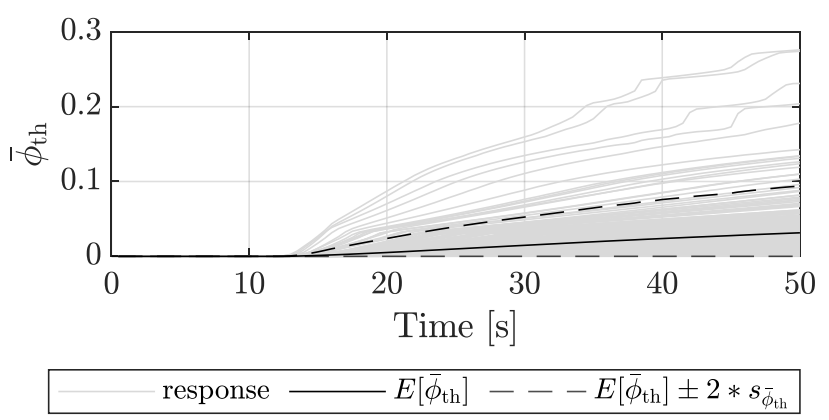

Fig. 5: Volume fraction of thrombosis. The continuous line shows the mean value; the dashed line represents the 2 standard deviations of the data.
Table 2: Sobol indices of the input random variables on the maximum volume fraction of thrombosis

\begin{tabular}{lccc}
\hline & $S_{\mathrm{i}}$ & $S_{\mathrm{i}}^{\mathrm{T}}$ & $S_{\mathrm{i}}^{\mathrm{T}}-S_{\mathrm{i}}$ \\
\hline$c_{\mathrm{BPt}}$ & 0.638 & 0.730 & 0.093 \\
$k_{\mathrm{BP}}$ & 0.198 & 0.277 & 0.078 \\
$c_{\mathrm{AP}}$ & 0.043 & 0.060 & 0.017 \\
$D_{\mathrm{c}}$ & 0.012 & 0.017 & 0.004 \\
$T_{\mathrm{R}}$ & 0.007 & 0.014 & 0.007 \\
$k_{\mathrm{c}, \text { wall }}$ & 0.001 & 0.008 & 0.007 \\
$c_{\mathrm{BPbt}}$ & 0.000 & 0.005 & 0.004 \\
$c_{\mathrm{t}}$ & 0.000 & 0.005 & 0.005 \\
$K_{\mathrm{c}}$ & 0.000 & 0.001 & 0.001 \\
\hline Total & 0.900 & 1.117 & 0.218 \\
\hline
\end{tabular}

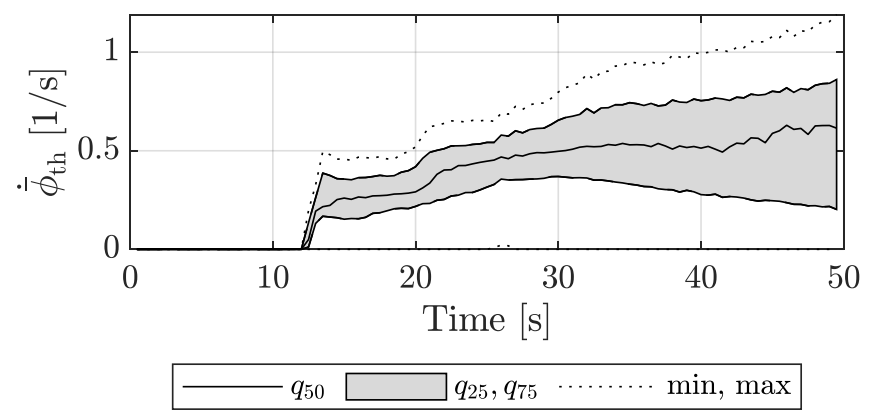

Fig. 6: Thrombus growth rate in time normalized by each maximum. The continuous black line identifies the median value; the grey area represents the interquartile range; the dotted lines are the maximum and minimum data points.

Since the thrombus develops in time, it is important to understand how the variation of its development evolves in time. The results show several trends (Fig. 5 and Fig. 6). Increasing variability in time is visible, especially towards the end of the simulation time. Such a trend is critical for a model in which the computation of the thrombus volume is the final goal. The results of the generalized Sobol indices for the volume fraction of thrombosis and the thrombus growth rate are shown in Fig. 7 and Fig. 8, respectively.

\section{Conclusions}

In Tab.2, the first- and total-order sensitivity indices and their differences are shown. The bounded platelets threshold $c_{\mathrm{BPt}}$ accounts alone, i.e. without considering interactions, for about $64 \%$ of the variation of the volume fraction of thrombosis. By subtracting the first-order from the total-order indices of Tab. 2, the interaction effect is estimated. The input $c_{\mathrm{BPt}}$ expresses a relatively high interaction level, which suggests that not only the bounded platelets threshold $c_{\mathrm{BPt}}$ should be carefully defined in the model, but also that its interaction with other input factors has to be investigated.

Input parameters such as the bounded platelets reaction rate $k_{\mathrm{BP}}$ and the concentration of activated platelets $c_{\mathrm{AP}}$ also, show high sensitivity indices, and therefore their proper determination requires further investigation. In general, the input parameters that include the platelet's mechanics control most of the process, except the bounded platelets 
threshold at the boundary $c_{\mathrm{BPbt}}$. All the other input random variables, including the parameters relating to the coagulant, have little to no influence on the considered output, and therefore they could be switched to fixed values without altering the model response.

The sensitivity analysis in time of the volume fraction of thrombosis and the growth rate also shows the big influence influence of the bounded platelets threshold $c_{\mathrm{BPt}}$, Fig. 7 and 8. Of interest is the role of the residence time threshold $T_{\mathrm{R}}$ in the early stage of thrombus formation. Its primary influence at sparking off the formation of the thrombus is visible. This behaviour is expected and mimics the physical condition for thrombus formation. The influence of the coagulant diffusivity $D_{\text {c }}$ presents a peak right after the first formation of the thrombus, meaning that it is guiding the formation where the coagulant diffuse better. Successively, its influence decreases to a level of little significance. The time-dependent influences of all other input random variables confirm the result obtained for the volume fraction of thrombosis and can be considered as constant.

In conclusion, the results of the sensitivity analysis for the model outputs show that the bounded platelets threshold $c_{\mathrm{BPt}}$ exerts the most substantial influence. The reaction rate of bounded platelets $k_{\mathrm{BP}}$ plays the second-largest role in the thrombus growth rate and the volume fraction of thrombosis.
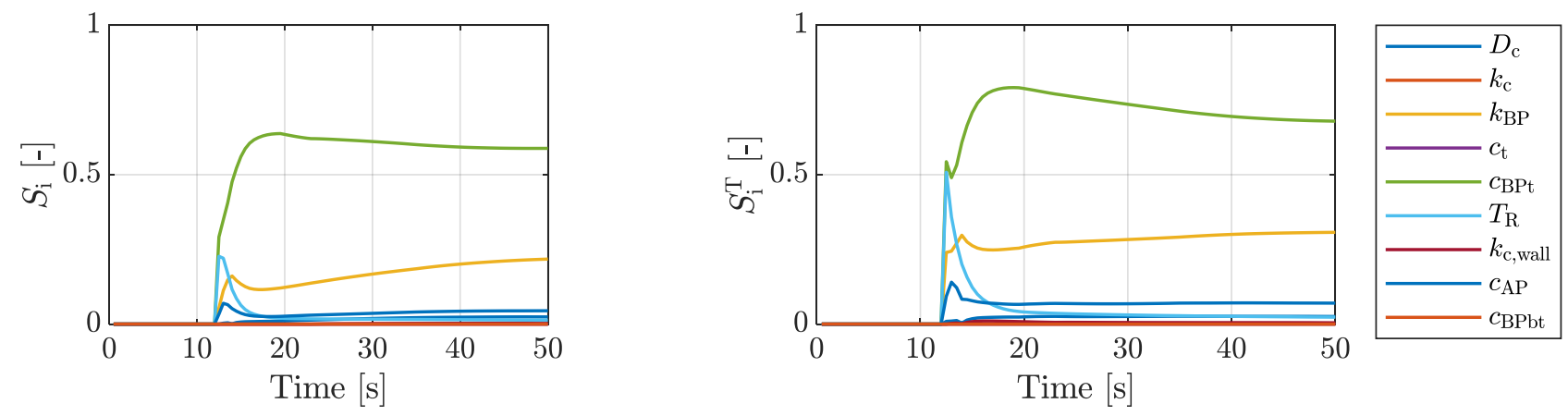

Figure 4: Generalized first- (left) and total-Sobol (right) indices for the volume fraction of thrombosis.
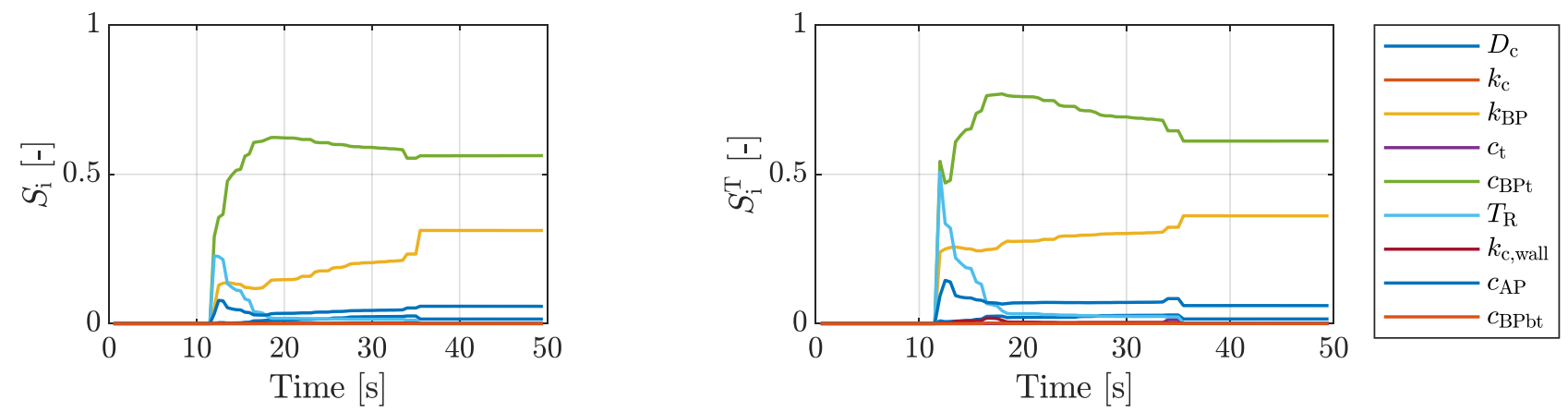

Fig. 5: Generalized first- (left) and total-Sobol (right) indices for the growth rate of the thrombus.

\section{Acknowledgements}

The Graz University of Technology supports this work through the LEAD Project "Mechanics, Modeling, and Simulation of Aortic Dissection" and by the Graz Center of Computational Engineering.

\section{References}

[1] C.A. Nienaber, R.E. Clough, N. Sakalihasan, T. Suzuki, R. Gibbs, F. Mussa, M.P. Jenkins, M.M. Thompson, A. Evangelista, J.S.M. Yeh, N. Cheshire, U. Rosendahl \& J. Pepper “Aortic dissection". In: Nature Reviews Disease Primers 2.1 (2016), pp. 1-18. 
[2] C. Menichini and X.Y. Xu. "Mathematical modeling of thrombus formation in idealized models of aortic dissection: initial findings and potential applications". In: Journal of mathematical biology 73.5 (2016), pp. 1205-1226.

[3] T.T. Tsai, A. Evangelista, C.A. Nienaber, T. Myrmel, G. Meinhardt, J.V. Cooper, D.E. Smith, T. Suzuki, R. Fattori, A. Llovet, J. Froehlich, S. Hutchison, A. Distante, T. Sundt, J. Beckman, J.L. Januzzi, E.M. Isselbacher, K.A. Eagle. "Partial thrombosis of the false lumen in patients with acute type B aortic dissection". In: I 357.4 (2007), pp. $349-359$.

[4] Y. Bernard, H. Zimmermann, S. Chocron, J-F. Litzler, B. Kastler, J-P. Etievent, N. Meneveau, F. Schiele, J-P. Bassand. "False lumen patency as a predictor of late outcome in aortic dissection". In: The American journal of cardiology 87.12 (2001), pp. 1378-1382.

[5] S. Trimarchi, J.L. Tolenaar, F.H.W. Jonker, B. Murray, T.T. Tsai, K.A.Eagle, V. Rampoldi, H.J.M. Verhagen, J.A.van Herwaarden, F.L. Moll, B.E. Muhs, J.A. Elefteriades Importance of false lumen thrombosis in type B aortic dissection prognosis. 2013.

[6] C. Menichini, Z.Cheng, R.G.J. Gibbs and X.Y. Xu "Predicting false lumen thrombosis in patients-specific models of aortic dissection". In: Journal of The Royal Society Interface 13.124 (2016), p. 20160759.

[7] B. Sudret. "Global sensitivity analysis using polynomial chaos expansions". In: Reliability engineering \& system safety 93.7 (2008), pp. 964-979.

[8] J.O. Taylor, K.P. Witmer, T. Neuberger, B.A. Craven, R.S. Meyer, S. Deutsch, K.B. Manning "In vitro quantification of time dependent thrombus size using magnetic resonance imaging and computational simulations of thrombus surface shear stresses". In: Journal of biomechanical engineering 136.7 (2014).

[9] J.O. Taylor, R.S. Meyer, S. Deutsch, K.B. Manning "Development of a computational model for macroscopic predictions of device-induced thrombosis”. In: Biomechanics and modeling in mechanobiology 15.6 (2016), pp. 17131731.

[10] I. M. Sobol. "Global sensitivity indices for nonlinear mathematical models and their Monte Carlo estimates". In: Mathematics and computers in simulation 55.1-3 (2001), pp. 271-280.

[11] R. G. Ghanem, P. D. Spanos. Stochastic Finite elements: a spectral approach. Courier Corporation, 2003.

[12] D. Xiu and G. E. Karniadakis. "The Wiener-Askey polynomial chaos for stochastic differential equations". In: SIAM journal on scientific computing 24.2 (2002), pp. 619-644.

[13] T. Crestaux, O. Le Maître, and J. Martinez. "Polynomial chaos expansion for sensitivity analysis". In: Reliability Engineering \& System Safety 94.7 (2009), pp. 1161-1172.

[14] A. Alexanderian, P. A. Gremaud, and R. C. Smith. "Variance-based sensitivity analysis for time-dependent processes". In: Reliability Engineering \& System Safety, 196 (2020), pp. 106722.

[15] S. Marelli and B. Sudret. "UQLab: A framework for uncertainty quantification in Matlab". In: Vulnerability, uncertainty, and risk: quantification, mitigation, and management. 2014, pp. 2554-2563. 